\title{
Robust Synchronization via Homogeneous Parameter-dependent Polynomial Contraction Matrix
}

\author{
Dongkun Han and Graziano Chesi
}

\begin{abstract}
Robust synchronization problem is a key issue in chaotic circuits and nonlinear systems. This paper is concerned with robust synchronization problem of polynomial nonlinear system affected by time-varying uncertainties on topology, i.e., structured uncertain parameters constrained in a boundedrate polytope. Via partial contraction analysis, novel conditions, both for robust exponential synchronization and for robust asymptotical synchronization, are proposed by using parameterdependent contraction matrices. In addition, for polynomial nonlinear system, this paper introduces a new class of contraction matrix, i.e., homogeneous parameter-dependent polynomial contraction matrix (HPD-PCM), by which tractable conditions of linear matrix inequalities (LMIs) are provided via affine space parametrizations. Furthermore, the variant rate margin for robust asymptotical synchronization is, for the first time, proposed and investigated via handling generalized eigenvalue problems (GEVPs). A set of representative examples demonstrate the effectiveness of proposed method.
\end{abstract}

\section{INTRODUCTION}

In the past decade, synchronization problem of chaotic systems and complex dynamical networks has been an active topic due to its broad applications in widespread academic fields since the pioneering work of Pecora and Carroll [1]. Indeed, complex networks have been testified as useful and powerful tools for modeling a great deal of chaotic circuits and other real-world systems which otherwise appear intractable [2]. Another related and well-known issue of multi-agent systems is the consensus problem, which, very interestingly, shares common features with synchronization problem of complex networks. Common examples exist in World Wide Web, electrical power grid and biological Metabolism [3]-[5].

Synchronization of coupled networks is extensively investigated for fixed topology by first Lyapunov method and Lyapunov exponents. Both methods can generate rigorous stability conditions where the former one needs to construct a suitable Lyapunov function, while the latter one, by local linearization and by block diagonalizing vector fields, the maximum Lyapunov exponents of complex system is introduced to ensure the synchronization manifold to be locally transversal stable [6]. Then, under miscellaneous assumptions, local synchronization conditions of linearizable system are provided based on wide variety of criteria [7]. As a different approach which casts special attention to the evolution of trajectories, the contraction theory has been brought in the convergence analysis of nonlinear system and also has been

Copyright (c) 2014 IEEE. Personal use of this material is permitted. However, permission to use this material for any other purposes must be obtained from the IEEE by sending an email to pubs-permissions@ieee.org

Both authors are with the Department of Electrical and Electronic Engineering, The University of Hong Kong, Pokfulam Road, Hong Kong. E-mail dkhan@eee.hku.hk. identified as an effective tool for analyzing the exponential synchronization of complex networks [8], [9].

However, real-world is overflowing with perturbations and disturbances. For a simple instance in electrical power grid, the basic parameters of transmission lines, such as the values of resistance and capacitance, are vulnerable to change under different temperature and air pressure, displaying uncertainties from time to time. Thus, numerous attentions have recently been paid to robust synchronization of complex systems with uncertainties and time-varying topology [10]-[14]. In [10], by searching a Quadratic Lyapunov Functions (QLF), robust synchronization conditions are provided for uncertain system whose control gains are disturbed by square integrable bounded time-varying uncertainties. In [11], impulsive synchronization criteria is proposed for uncertain dynamical network where the network coupling functions are unknown but bounded, under assumptions that both the intrinsic nonlinear function and the coupling nonlinear function satisfy Lipschitz-like conditions. In [12], for fast-switching topology, a local synchronization criteria is given at a sufficiently large switching rate. Also for local synchronization, conditions are proposed by using the time-average topology to approximate the time-varying topology [13]. By using contraction theory, polynomial system with time-invariant uncertainty is considered where robust stability is established via searching a parameter-independent polynomial contraction matrix [14].

Time-varying networks can also be modelled by stochastic switching networks [15]-[19]. In [15], a connection graph stability method, also proposed by [20], is extended to a blinking model of small-world network in which both fixed $2 K$-nearest neighbor coupling and time-dependent on-off coupling are considered. In [16], each agent is assumed to be a random walker and random changes of network can be described by the change of agents' locations in the lattice where information can be transmitted only for agents in the same lattice. In [17], sufficient conditions are proposed for global synchronization with a fast and random switching network by using stochastic Lyapunov stability theory. In [18], [19], a stochastically blinking system is considered where topological parameters randomly switch within a discrete set of values at a same time intervals.

The motivations of this paper rest with the facts that firstly, robust synchronization with time-varying uncertainty desirably meets the demand of practical implements and has already been successfully applied in wireless sensor networks and neural networks [21]. In addition, existing uncertain models for synchronization protocols usually assume that there exists time-invariant uncertainty or slowly time-varying uncertainty, thus making special academic interests in time-varying uncertainty with bounded variation rate (See Subsection II- 
B). Furthermore, for adjacency matrix perturbed by uncertain parameters, traditional approaches like eigenvalue analysis are in an extremely difficulty to apply, while it can be suitably tacked with parameter-dependent contraction theory. Last but not the least, even comparing with the prevailing approaches, the QLFs method or parameter-dependent Lyapunov method, the parameter-dependent contraction analysis maintains its advantages in that it does not require an error dynamics (whose construction needs additional assumptions or approximations), and in some circumstances makes the Lyapunov methods as special cases (See Subsection III-A).

Based on the motivations aforementioned, and contrast with the literatures, this paper considers robust exponential synchronization and robust asymptotical synchronization problems affected by time-varying topological uncertainty with bounded variation rate via parameter-dependent contraction analysis. The contributions of this paper are listed as below. (1) For the first time, to the best of our knowledge, the timevarying topological uncertainty with bounded variation rate is considered in robust synchronization problem, making the case with time-invariant uncertainty and the case with time-varying polytopic uncertainty as special ones. (2) An approach of parameter-dependent contraction matrix is proposed by using a general infinitesimal length, which is less conservative than the cases using constant contraction matrix or Lyapunov-like approach. (3) Distinct with nonlinear inequalities provided by traditional methods, this paper provides tractable conditions of LMIs for robust synchronization problem by employing SMR and by parametrizing suitable affine spaces. (4) For robust asymptotical synchronization, the lower bound of variation rate margin is estimated via handling GEVPs.

\section{Preliminaries}

Notations: $\mathbb{N}, \mathbb{R}$ : natural and real number sets; $\mathbf{0}_{n}$ : origin of $\mathbb{R}^{n} ; \mathbb{R}_{0}^{n}: \mathbb{R}^{n} \backslash\left\{\mathbf{0}_{n}\right\} ; A^{T}$ : transpose of $A ; A>0(A \geq 0)$ : symmetric positive definite (semidefinite) matrix $A ; I_{n}: n \times n$ identity matrix; $A \otimes B$ : Kronecker product of matrices $A$ and $B ; A^{\mathrm{s}}: \frac{1}{2}\left(A+A^{T}\right)$, with $A \in \mathbb{R}^{n \times n} ; \operatorname{co}\left\{X_{1}, \ldots, X_{p}\right\}:$ convex hull of matrices $X_{1}, \ldots, X_{p} \in \mathbb{R}^{m \times n} ; x^{i}: x_{1}^{i_{1}} x_{2}^{i_{2}} \cdots x_{n}^{i_{n}}$, $x \in \mathbb{R}^{n}, i \in \mathbb{N}^{n} ; \operatorname{sq}(\theta):\left(\theta_{1}^{2}, \ldots, \theta_{a}^{2}\right)^{T} \in \mathbb{R}^{a}, \theta \in \mathbb{R}^{a} ;(*)^{T} A B$ in a form of SMR: $B^{T} A B$.

Given a vector norm $|\cdot|_{i}$ on Euclidean space $(|\cdot|)$, with a induced matrix norm $\|A\|_{i}$ given by

$$
\|A\|_{i}=\max _{|x|_{i}=1} \frac{|A x|_{i}}{|x|_{i}}
$$

and the associated matrix measure $\mu_{i}$ is defined as the onesided directional derivative of $\|\cdot\|_{i}$ in the direction $A$, which is denoted by

$$
\mu_{i}(A):=\lim _{\epsilon \rightarrow 0} \frac{\left(\|I+\epsilon A\|_{i}-1\right)}{\epsilon} .
$$

\section{A. Basics of Contraction Theory}

To introduce contraction theory, let us consider a deterministic dynamical sysem of following time-dependent ordinary differential equation

$$
\dot{x}=f(x, t), x\left(t_{0}\right)=x_{0}, t_{0} \geq 0
$$

where $f$ is a nonlinear vector field and $x$ is a state vector in a subset of $\mathbb{R}^{n}$. Under the assumption that $f$ is continuously differentiable, one can obtain an exact differential relation

$$
\delta \dot{x}=J(x, t) \delta x
$$

where $J(x, t)=\frac{\partial f}{\partial x}$ denotes the Jacobian of the vector field $f$, and $\delta x$ denotes an infinitesimal change evaluated along a trajectory. $\delta x$ is also called "virtual displacement" pervasive in classical mechanics and formally considered as a linear tangent differential form with respect to time [8], [22].

Definition 1: (Contraction) System (1) is said to be contracting if there exists some $c>0$ such that for every two solutions $x(t)=\nu(t, 0, \xi)$ and $y(t)=\nu(t, 0, \zeta)$ of System (1), starting from different initial conditions, converge exponentially to each other, i.e. $|x(t)-y(t)| \leq e^{-c t}|\xi-\zeta|$ where $f(x, t)$ is called a contracting function.

Paralleling with above definition, another one is given here for global asymptotical contraction behavior.

Definition 2: (Asymptotical Contraction) System (1) is said to be asymptotically contracting if for every two solutions $x(t)=\nu(t, 0, \xi)$ and $y(t)=\nu(t, 0, \zeta)$ of System (1), starting from different initial conditions, converge asymptotically to each other, i.e. $\lim _{t \rightarrow \infty}|x(t)-y(t)|=0$ where $f(x, t)$ is called an asymptotically contracting function.

System (2) can be considered as a linear time-varying differential equation $\delta \dot{x}=J(t) \delta x$ where $J(t)$ is a function of time. One can obtain an upper bound for the magnitude of its solutions by the Coppel Inequality as follows [23],

$$
|\delta x|_{i} \leq\left|\delta x_{0}\right|_{i} e^{\int_{0}^{t} \mu_{i}(J(\tau)) d \tau}
$$

where $\mu(J)$ is the matrix measure of the Jacobian matrix of $f$. Following result displays an essential conclusion about contracting systems which can be tracked down from miscellaneous technical assumptions [8], [24].

Lemma 1: The system (1) is contracting if there exist some matrix measure $\mu_{i}(J(x, t))$ and a positive constant $c$ such that

$$
\mu_{i}(J(x, t)) \leq-c_{i}
$$

where the scalar $c_{i}$ denotes the contraction rate of the system corresponding to vector norm $|\cdot|_{i}$.

The matrix measure $\mu_{i}$ corresponding to the induced matrix norms $\|\cdot\|_{1},\|\cdot\|_{2}$ and $\|\cdot\|_{\infty}$ can be calculated and given in real domain [8], [25] and in complex domain [26]. However, for a particular vector norm and its associated induced matrix norm, it is in general a difficult task to obtain an explicit expression [25]. From following Proposition, a clue will be given on the relationship amongst different matrix measures about contraction. At first, let us introduce following Lemma.

Lemma 2 ([27]): For any two positive real numbers $p>$ $q>0$, considering a vector space $\mathscr{V}$ with finite dimension $n$ respect to vector norms $|\cdot|_{q}$ and $|\cdot|_{p}$, a relationship can be given by

$$
|x|_{p} \leq|x|_{q} \leq n^{(1 / q-1 / p)}|x|_{p} .
$$

Proposition 1: (Equivalence on contraction) For positive real numbers $p, q$ with $p>q>0,|\cdot|_{q}$ and $|\cdot|_{p}$ are two vector norms on $\mathscr{V}$, System 1 is contracting for vector norm 
$|\cdot|_{q}$ with contraction rate $c_{q}$, i.e., which implies that it is also contracting for vector norm $|\cdot|_{p}$ at the same contraction rate with a time-shift $\psi=\frac{(p-q) \log n}{\left(p q c_{q}\right)}$, i.e.,

$$
|\delta x|_{p} \leq\left|\delta x_{0}\right|_{p} e^{-c_{q}(t-\psi)} .
$$

Proof See Appendix A.

Considering the equivalence of contraction, we select Euclidean norm as [8] for ease of description and LMI relaxation.

\section{B. Problem Formulation}

In this paper, the uncertain multi-agent systems with timevarying topological uncertainties is considered under following synchronization protocol

$$
\dot{x}_{i}(t)=f\left(x_{i}(t)\right)-b \sum_{j=1}^{N} L_{i j}(\theta(t)) \Gamma x_{j}(t), \quad i, j=1, \ldots, N
$$

where $x_{i} \in \mathbb{R}^{n}$ is the state of $i$-th agent, $N$ is the number of agents, $b$ is the coupling weight, $f\left(x_{i}\right) \in \mathbb{R}^{n}$ is a nonlinear function, $\Gamma=\operatorname{diag}\left(\gamma_{1}, \ldots, \gamma_{n}\right) \in \mathbb{R}^{n \times n}$ is a diagonal matrix where $\gamma_{i}>0$ stands for the agents communicating through their $i$-th states. $\theta(t) \in \mathbb{R}^{a}$ denotes the time-varying perturbations from environment to the network topology. Let

$$
(\theta(t), \dot{\theta}(t)) \in \Omega=\left\{(\theta(t), \dot{\theta}(t)): \theta(t) \in \Lambda_{a}, \dot{\theta}(t) \in \Xi\right\}
$$

in which $\Lambda_{a}$ is a simplex and $\Xi$ is a polytope given by

$$
\left\{\begin{array}{l}
\Lambda_{a}=\left\{\theta(t) \in \mathbb{R}^{a}: \sum_{i=1}^{a} \theta_{i}(t)=1, \theta_{i}(t) \geq 0\right\} \\
\Xi=\operatorname{co}\left\{d^{(1)}, \ldots, d^{(v)}\right\}
\end{array}\right.
$$

for some given vectors $d^{(1)}, \ldots, d^{(v)} \in \mathbb{R}^{a}$ such that $\sum_{i=1}^{a} d_{i}^{(j)}=0, \forall j=1, \ldots, v$ and $0_{a} \in \Xi$ where $0_{a}$ is a column vector with all $a$ entries being zero. The model (7) has been introduced by [28] and is developed as an extension of models adopted in previous works [29], [30], including various famous models as special cases. $L_{i j}(\theta(t))$ is the $i j$ th entry of the uncertain Laplacian matrix $L(\theta(t)) \in \mathbb{R}^{N \times N}$ given by $L_{i j}(\theta(t))=-G_{i j}(\theta(t))$ for all $i \neq j$ and by $L_{i i}(\theta(t))=-\sum_{j=1, j \neq i}^{N} L_{i j}(\theta(t))$.

Remark 1: Synchronization protocol (6) is a general and applicable form. It generalises some typical models, e.g., synchronization protocol with time-invariant uncertainty applied in voltage analysis of chaotic circuits [2]. As a nonautonomous system with time-varying input, it implies that not merely moving equilibrium point is considered, but bounded manifolds like periodic orbit or chaotic oscillator.

Remark 2: Linear pertubation in communication network is widely adopted in literatures [10]. In this paper, we also assume $G_{i j}(\theta(t))$ is a linear function thus the uncertain Laplacian matrix can be expressed as

$$
L(\theta(t))=L_{0}+\sum_{i=1}^{a} \theta_{i}(t) L_{i}
$$

Nonlinear coupling with nonlinear perturbations will also be discussed in Section III.

Let us introduce the uncertain multi-agent dynamical system (6) in compact form as

$$
\dot{x}(t)=g(x(t))-b(L(\theta(t)) \otimes \Gamma) x(t)
$$

where $x(t)=\left(x_{1}(t)^{T}, \ldots, x_{N}(t)^{T}\right)^{T}$ and $g(x(t))=$ $\left(f\left(x_{1}(t)\right)^{T}, \ldots, f\left(x_{N}(t)\right)^{T}\right)^{T}$. Then, the robust synchronization problems can be proposed as follows:

Problem 1: To establish if the uncertain dynamical system (9) achieves robust global and exponential synchronization, i.e. for any $\epsilon$ there exist positive constants $\kappa$ and $c$ such that $\left\|x_{i}(t)-x_{j}(t)\right\| \leq \kappa\left\|x_{i}(0)-x_{j}(0)\right\| e^{-c t}$ for all $x_{i}(0), x_{j}(0)$, $\theta(t) \in \Omega$ and $i, j=1, \ldots, N$.

Problem 2: To establish if the uncertain dynamical system (9) achieves robust global and asymptotical synchronization, i.e. for any $\epsilon$ there exist $T(\epsilon)>0$ such that $\left\|x_{i}(t)-x_{j}(t)\right\| \leq \epsilon$ and $\lim _{t \rightarrow 0}\left\|x_{i}(t)-x_{j}(t)\right\|=0$ for all $t>T, x_{i}(0), x_{j}(0)$, $\theta(t) \in \Omega$ and $i, j=1, \ldots, N$.

\section{MAIN RESULTS}

In this section, the conception of parameter-dependent contractive matrix will be proposed and corresponding robust synchronization conditions will be established via the partial contraction and SMR technique.

\section{A. Robust Synchronization Conditions}

An easy yet effective way to analyze synchronization without topological uncertainty via contraction theory is the method of partial contraction, where an auxiliary system is introduced and the desired convergence behaviour is isolated from the overall system dynamics [9].

Lemma 3 ([9]): Consider a continuously differentiable nonlinear system of the form $\dot{x}=f(x, x, t)$ and there exists an auxiliary system $\dot{y}=f(y, x, t)$ which is contracting with respect to $y$. If a particular solution of the auxiliary $y$-system verifies a smooth specific property, then all trajectories of the original $x$-system verify this property exponentially. The original system is said to be partially contracting.

One can observe that the virtual system ( $y$-system) has two particular solutions, i.e., $y(t)=x(t)$ sharing the specific property. If all trajectories of virtual system converge exponentially to a specific trajectory, it directly implies $x(t)$ exponentially verifies these properties.

Example 1: Let us consider a synchronization problem via using partial contraction. Given a pair of unidirectional coupled oscillators as follows:

$$
\left\{\begin{array}{l}
\dot{x_{1}}=f\left(x_{1}, t\right) \\
\dot{x_{2}}=f\left(x_{2}, t\right)+u\left(x_{1}\right)-u\left(x_{2}\right)
\end{array}\right.
$$

where $x_{1}, x_{2} \in \mathbb{R}^{n}$ are state vectors, $f\left(x_{i}, t\right)$ is the dynamics of uncoupled oscillators and $u\left(x_{1}\right)-u\left(x_{2}\right)$ denotes the coupling force. We can select a virtual system

$$
\dot{y}=f(y, t)-u(y)+u\left(x_{1}\right) \text {. }
$$

It is obvious that $x_{1}(t)=x_{2}(t)$ is a particular solution. On the condition that $f-u$ is contracting, synchronization can be achieved exponentially.

Definition 3: Let $\dot{y}=h(y, \theta, t)$ be the auxiliary system of (6), $M(y, \theta)$ is defined to be a parameter-dependent contraction matrix (PD-CM) which is symmetric and uniformly positive definite such that

$$
\left(2 \frac{\partial h^{T}}{\partial y} M+\frac{\partial M}{\partial \theta} \dot{\theta}+\frac{\partial M}{\partial y} \dot{y}\right)^{\mathrm{s}} \leq-\gamma M
$$


where $\gamma$ is a strictly positive scalar. Similarly, $M(y, \theta)$ is called parameter-dependent asymptotical contraction matrix which is symmetric and uniformly positive definite such that

$$
\left(2 \frac{\partial h^{T}}{\partial y} M+\frac{\partial M}{\partial \theta} \dot{\theta}+\frac{\partial M}{\partial y} \dot{y}\right)^{\mathrm{s}} \leq-\gamma I
$$

where $\gamma$ is a strictly positive scalar.

Lemma 4 ([31]): Let $A \in \mathbb{R}^{N \times N}$ be a symmetric matrix. Product matrix $W_{A}^{N}=\left(1_{N} \cdot 1_{N}^{T}\right) \otimes A$ is positive semidefinite if and only if $A \geq 0$, where $1_{N}$ is a column vector with all entries being one.

Theorem 1: Consider an uncertain system (9), an auxiliary system can be obtained as

$$
\begin{aligned}
\dot{y}(t)= & g(y(t))-b(L(\theta(t)) \otimes \Gamma) y(t) \\
& -W_{P \Gamma}^{N}(\theta) y(t)+W_{P \Gamma}^{N}(\theta) x(t)
\end{aligned}
$$

where $W_{P \Gamma}^{N}(\theta)=\left(1_{N} \cdot 1_{N}^{T}\right) \otimes(P(\theta) \cdot \Gamma)$ and $P(\theta) \in \mathbb{R}^{n \times n}$ is a positive definite matrix for all $(\theta(t), \dot{\theta}(t)) \in \Omega$. Furthermore, robust global exponential synchronization can be achieved if there exists a parameter-dependent contraction matrix $M(y, \theta)$ such that $g(y(t))-b(L(\theta(t)) \otimes \Gamma) y(t)$ is contracting.

Proof By introducing a positive semidefinite matrix $P(\theta)$, for $i, j=1, \ldots, N,(6)$ can be equivalently expressed as

$$
\begin{aligned}
\dot{x}_{i}(t)= & f\left(x_{i}(t)\right)-b \sum_{j=1}^{N} L_{i j}(\theta(t)) \Gamma x_{j}(t) \\
& -P(\theta) \sum_{j=1}^{N} \Gamma x_{j}+P(\theta) \sum_{j=1}^{N} \Gamma x_{j} .
\end{aligned}
$$

Then one can obtain a compact form similarly from (9) such that

$$
\begin{aligned}
\dot{x}(t)= & g(x(t))-b(L(\theta(t)) \otimes \Gamma) x(t) \\
& -W_{P \Gamma}^{N}(\theta) x(t)+W_{P \Gamma}^{N}(\theta) x(t) .
\end{aligned}
$$

Thus, by considering $W_{P \Gamma}^{N}(\theta) x(t)$ as the system inputs, the auxiliary system (13) can be obtained that a particular solution of robust synchronization is $y^{*}=1_{N} \otimes y_{\infty}$ where

$\dot{y}_{\infty}(t)=f\left(y_{\infty}\right)-N P \Gamma y_{\infty}+P(\theta) \sum_{j=1}^{N} \Gamma x_{j}, i, j=1, \ldots, N$.

Considering Lemma 3, the robust synchronization of system (9) for all $(\theta(t), \dot{\theta}(t)) \in \Omega$ can be achieved and the property $x_{1}=\ldots=x_{N}$ can be verified exponentially if system (13) is contracting. Thus, (13) is an auxiliary system for system (9).

Next, we will show that the auxiliary system (13) is contracting if there exists a parameter-dependent contraction matrix. A concise proof of exponential convergence of trajectories for contracting system is given in [32] for an uncertainty-free case. Let $y_{0}$ and $y_{1}$ be two different points and let $\Upsilon(y, \theta, t)$ be the associated flow of the auxiliary system (13). If there exists a parameter-dependent contraction matrix $M(y, \theta)$ given by Definition 3, then by the Theorem 2 of [32] one can obtain

$$
D_{M}\left(\Upsilon\left(y_{0}, \theta, t\right), \Upsilon\left(y_{1}, \theta, t\right)\right) \leq e^{(-c / 2) t} D_{M}\left(y_{0}, y_{1}\right),
$$

where $D_{M}$ is the geodesic distance corresponding to the metric $M(y, \theta)$. Here mapping $\Upsilon$ is a strict contraction. Then, according to Contraction Mapping Theorem, the flow $\Upsilon(y, \theta, t)$ verifies a specific manifold $y_{\infty}(t)$ exponentially [33].

Lastly it will be shown that there exists a parameterdependent contraction matrix $M$ such that (13) is contracting.
Since $g(y(t))-b(L(\theta(t)) \otimes \Gamma) y(t)$ is contracting, one has that there exists a matrix $M(\theta)$ such that

$$
\begin{aligned}
& 2\left(\frac{\partial h^{T}}{\partial y} \tilde{M}+\frac{\partial \tilde{M}}{\partial \theta} \dot{\theta}+\frac{\partial \tilde{M}}{\partial y} \dot{y}\right)^{\mathrm{s}} \\
= & \left.2\left(\frac{\partial g^{T}}{\partial y} \tilde{M}\right)^{\mathrm{S}}-2 b((L(\theta)) \otimes \Gamma)^{T} \tilde{M}\right)^{\mathrm{S}} \\
& +\left(\frac{\partial \tilde{M}}{\partial \theta} \dot{\theta}\right)^{\mathrm{S}}+\left(\frac{\partial \tilde{M}}{\partial y} \dot{y}\right)^{\mathrm{s}} \\
\leq & -\gamma \tilde{M} .
\end{aligned}
$$

Since $\Gamma$ is diagonal positive semidefinite and $P(\theta)$ is a positive semidefinite matrix, one has that $W_{P \Gamma}^{N}(\theta)^{\mathrm{s}} \geq 0$ by Lemma 4 . Thus, the Riemanian manifold of general infinitesimal length for the auxiliary system (13) can be expressed by

$$
\begin{aligned}
& \frac{\mathrm{d}}{\mathrm{d} t} \delta y^{T} \tilde{M}(\theta, y) \delta y \\
= & \frac{\mathrm{d}}{\mathrm{d} t} \delta y^{T} \tilde{M}(\theta, y)^{\mathrm{s}} \delta y \\
= & \delta y^{T}\left(\left(2 \frac{\partial h^{T}}{\partial y} \tilde{M}\right)^{\mathrm{s}}+\left(\frac{\partial M}{\partial \theta} \dot{\theta}\right)^{\mathrm{s}}+\left(\frac{\partial \tilde{M}}{\partial y} \dot{y}\right)^{\mathrm{s}}\right) \delta y \\
= & \delta y^{T}\left(2\left(\frac{\partial g^{T}}{\partial y} \tilde{M}\right)^{\mathrm{s}}-2 b((L(\theta)) \otimes \Gamma)^{T} \tilde{M}\right)^{\mathrm{s}} \\
& \left.-2\left(\left(W_{P \Gamma}^{N}(\theta)\right)^{T} \tilde{M}\right)^{\mathrm{s}}+\left(\frac{\partial M}{\partial \theta} \dot{\theta}\right)^{\mathrm{s}}+\left(\frac{\partial \tilde{M}}{\partial y} \dot{y}\right)^{\mathrm{s}}\right) \delta y \\
= & \delta y^{T}\left(2\left(\frac{\partial g^{T}}{\partial y} \tilde{M}\right)^{\mathrm{s}}-2 b((L(\theta)) \otimes \Gamma)^{T} \tilde{M}\right)^{\mathrm{s}} \\
& \left.+\left(\frac{\partial M}{\partial \theta} \dot{\theta}\right)^{\mathrm{s}}+\left(\frac{\partial \tilde{M}}{\partial y} \dot{y}\right)^{\mathrm{s}}\right) \delta y-\delta y^{T}\left(\left(W_{P \Gamma}^{N}(\theta)\right)^{T} \tilde{M}\right) \delta y \\
& -\delta y^{T}\left(\tilde{M} W_{P \Gamma}^{N}(\theta)\right) \delta y \\
\leq & \delta y\left(2\left(\frac{\partial g^{T}}{\partial y} \tilde{M}\right)^{\mathrm{s}}-2 b((L(\theta)) \otimes \Gamma)^{T} \tilde{M}\right)^{\mathrm{s}} \\
& \left.+\left(\frac{\partial M}{\partial \theta} \dot{\theta}\right)^{\mathrm{s}}+\left(\frac{\partial \tilde{M}}{\partial y} \dot{y}\right)^{\mathrm{s}}\right) \delta y \\
\leq & -\gamma \delta y^{T} \tilde{M}(\theta, y) \delta y .
\end{aligned}
$$

Therefore, the auxiliary system is contracting which completes this proof.

A result can also be obtained for robust asymptotical synchronization by using parameter-dependent asymptotical contraction matrix as follows:

Theorem 2: Consider an uncertain system (9), an auxiliary system can be obtained as (13). Furthermore, robust asymptotical synchronization can be achieved if there exists a parameterdependent asymptotical contraction matrix $M(y, \theta)$ such that $g(y(t))-b(L(\theta(t)) \otimes \Gamma) y(t)$ is asymptotical contracting.

Proof Similar lines can be displayed in proof of Theorem 1 .

\section{Remark 3: For Theorem 1 and Theorem 2, note that}

- The virtual quantity of matrix $P(\theta)$ is to construct the auxiliary system (13), satisfying $\left(W_{P \Gamma}^{N}(\theta)^{T} \tilde{M}\right)^{\mathrm{S}} \geq 0$. Note that it has no influence on the actual systems, neither on the specific robust synchronization manifold nor on the robust synchronization rate. Moreover, matrix $P(\theta)$ in the auxiliary system is not unique.

- From Lemma 1, a more general case can be derived by using non-Euclidean norms and defining a general parameter-dependent contraction matrix such that

$$
\left\{\begin{array}{c}
M(y, \theta)=M(y, \theta)^{T} \geq 0, \forall \theta \in \Omega . \\
\frac{\mathrm{d}}{\mathrm{d} t}|M(y, \theta) \delta y|_{i} \leq-c_{i}|M(y, \theta) \delta y|_{i} .
\end{array}\right.
$$


B. Homogenous Parameter-dependent Polynomial Contraction Matrix

Establishing conditions of Theorem 1 and Theorem 2 is of great difficulties in that they are nonlinear inequality problems with time-varying uncertainties. However, via suitable parametrizations of affine spaces, SMR technique gives an effective way to solve these problems which amounts to tackling with an LMI feasibility test. Indeed, by introducing a new class of contraction matrix, i.e., HPD-PCM, robust synchronization conditions can be provided via solving an LMI feasibility test.

In this paper, we are interested in investigating the robust synchronization problems of polynomial nonlinear system. Thus before proceeding, let us introduce the following assumption on $f(x)$.

Assumption 1: The function $f\left(x_{i}\right)$ in (6) is polynomial.

Remark 4: An one-side global Lipschitz condition (or QUAD condition) is assumed in an overwhelming number of existing approaches for global synchronization such as [34]. However, the QUAD condition is not satisfied for simple nonlinearities such as quadratic and cubic functions. Instead, Assumption 1 includes such nonlinearities, and also includes important systems such as Lorenz-like system, Hamiltonian systems, Guckenheimer system and Rössler system.

Then, let us introduce the definition of homogeneous parameter-dependent polynomial as follows:

$$
m(y, \theta)=\sum_{\substack{q \in \mathbb{N}^{n} \\
r \in \mathbb{N}^{a}, \sum_{\begin{subarray}{c}{i=1 \\
i=1} }}^{a} q_{i}=2 d_{y}}\end{subarray}} c_{q, r} y^{q} \theta^{r},
$$

where $c_{q, r} \in \mathbb{R}$ is the coefficients of monomial $y^{q} \theta^{r}, d_{\theta}$ of $m(y, \theta)$ is the degree in $a$ scalar variables $\theta, 2 d_{y}$ of $m(y, \theta)$ is the degree in $\tilde{n}$ scalar variables $y$ and $\tilde{n}=N n$. Thus, a set of homogeneous parameter-dependent polynomial can be given as $\mathscr{H}=\{m(y, \theta):(15)$ holds $\}$. Then, the definition of HPD-PCM can be provided as

Definition 4: $M(y, \theta)$ is a HPD-PCM if it is a PD-CM and every entry of $M(y, \theta)$ satisfies

$$
M_{i j}(y, \theta) \in \mathscr{H}, \forall i, j=1, \ldots, \tilde{n} .
$$

Similarly, homogeneous parameter-dependent polynomial asymptotical contraction matrix (HPD-PACM) can be defined by using condition (12). Let $R(y, \theta, \dot{\theta}, \gamma)$ be a matrix of polynomial as

$$
\begin{aligned}
& R(y, \theta, \dot{\theta}, \gamma) \\
= & 2\left(\sum_{i=1}^{a} \theta_{i}\right)\left(\frac{\partial g^{T}}{\partial y} M\right)^{\mathrm{s}}-2 b\left((L(\theta) \otimes \Gamma)^{T} M\right)^{\mathrm{s}} \\
& +\left(\sum_{i=1}^{a} \theta_{i}\right)^{2}\left(\frac{\partial M^{T}}{\partial \theta} \dot{\theta}\right)^{\mathrm{s}}+\left(\sum_{i=1}^{a} \theta_{i}\right)\left(\frac{\partial M^{T}}{\partial y} g\right)^{\mathrm{s}} \\
& -b\left(\frac{\partial M}{\partial y}(L(\theta) \otimes \Gamma) y\right)^{\mathrm{s}}+\gamma\left(\sum_{i=1}^{a} \theta_{i}\right) M .
\end{aligned}
$$

Thus, condition (11) can be expressed in a homogeneous form of degree $d_{\theta}+1$ in $\theta$ since $\sum_{i=1}^{a} \theta_{i}=1$ for all $\theta \in \Omega$. The condition that $\theta \in \Lambda_{a}$ can be relaxed to the condition $\theta \in \mathbb{R}_{0}^{a}$ by the following lemma.
Lemma 5 ([35]): The function $H(\theta): \mathbb{R}^{a} \rightarrow \mathbb{R}^{n \times n}$ is a symmetric matrix consisted of homogenous polynomials with degree $d_{\theta}$ in $a$ scalar variables. Then,

$$
H(\theta)>0 \forall \theta \in \Lambda_{a} \Longleftrightarrow H(\operatorname{sq}(\theta))>0 \forall \theta \in \mathbb{R}_{0}^{\mathrm{a}} .
$$

Lemma 6: Robust exponential synchronization of (6) can be achieved under Assumption 1 if there exists a positive scalar $\gamma$ and a HPD-PCM $M(y, \theta)$ such that

$$
\left\{\begin{array}{l}
0<M(y, \mathrm{sq}(\theta)) \forall \mathrm{y} \in \mathbb{R}_{0}^{\tilde{n}}, \forall \theta \in \mathbb{R}_{0}^{\mathrm{a}} \\
0>R(y, \mathrm{sq}(\theta), \dot{\theta}, \gamma) \forall \mathrm{y} \in \mathbb{R}_{0}^{\tilde{n}}, \forall(\theta, \dot{\theta}) \in \Omega .
\end{array}\right.
$$

Proof This result can be obtained directly from Definition 3, Theorem 1 and Lemma 5. by

By the technique of SMR, $M(y, \mathrm{sq}(\theta))$ can be expressed

$$
M(y, \operatorname{sq}(\theta))=\Psi\left(\bar{M}, d_{y}, d_{\theta}, \tilde{n}\right)
$$

where

$\Psi\left(\bar{M}, d_{y}, d_{\theta}, \tilde{n}\right)=(*)^{T} \bar{M}\left(\phi_{\mathrm{pol}}\left(y, d_{y}\right) \otimes \phi_{\mathrm{hom}}\left(\theta, d_{\theta}\right) \otimes I_{\tilde{n}}\right)$,

$\phi_{\text {pol }}\left(y, d_{y}\right) \in \mathbb{R}^{l_{\text {pol }}\left(\tilde{n}, d_{y}\right)}$ is a power vector containing all monomials of degree less or equal to $d_{y}, \phi_{\text {hom }}\left(\theta, d_{\theta}\right) \in$ $\mathbb{R}^{l_{\text {hom }}\left(a, d_{\theta}\right)}$ is a power vector containing all monomial of degree $d_{\theta}$, and

$$
l_{\mathrm{pol}}\left(\tilde{n}, d_{y}\right)=\frac{\left(\tilde{n}+d_{y}\right) !}{\tilde{n} ! d_{y} !}, l_{\mathrm{hom}}\left(a, d_{\theta}\right)=\frac{\left(a+d_{\theta}-1\right) !}{(a-1) ! d_{\theta} !} .
$$

Symmetric matrix $\bar{M}$ belongs to the set

$$
\begin{aligned}
\mathscr{M}= & \left\{\bar{M}^{T}=\bar{M}: \Psi\left(\bar{M}, d_{y}, d_{\theta}, \tilde{n}\right)\right. \text { only contains } \\
& \text { monomials } \left.\theta^{i} \text { with even power } i_{k}\right\} .
\end{aligned}
$$

Lemma 7: The set $\mathscr{M}$ is a linear space of dimension

$$
\begin{aligned}
& \sigma\left(\tilde{n}, d_{y}, d_{\theta}\right)= \\
& \frac{1}{2} \tilde{n}\left(l_{\mathrm{pol}}\left(\tilde{n}, d_{y}\right) l_{\mathrm{hom}}\left(a, d_{\theta}\right)\left(\tilde{n} l_{\mathrm{pol}}\left(\tilde{n}, d_{y}\right) l_{\mathrm{hom}}\left(a, d_{\theta}\right)+1\right)\right. \\
& \left.-(\tilde{n}+1)\left(l_{\mathrm{hom}}\left(a, 2 d_{\theta}\right)-l_{\mathrm{hom}}\left(a, d_{\theta}\right)\right) l_{\mathrm{pol}}\left(\tilde{n}, 2 d_{y}\right)\right)
\end{aligned}
$$

Proof See Appendix B.

Thus, one can obtain a complete parametrization of the affine space $\mathscr{M}$ for HPD-PCM of (17). Now let us consider the SMR of $R(y, \theta, \dot{\theta}, \gamma)$. Note that the degree of polynomial $g(y)$ is $d_{g}$ in $y$ and define that

$$
d_{r}=\max \left(d_{g}-1+2 d_{y}, 2 d_{y}-1+d_{g}, 2 d_{y}\right),
$$

and $2 \tilde{d}_{r}=$ even $_{+1}\left(d_{r}\right)$ (i.e., $2 \tilde{d}_{r}=d_{r}$ if $d_{r}$ is even, and $2 \tilde{d}_{r}=d_{r}+1$ if $d_{r}$ is odd). It follows that,

$$
R(y, \theta, \dot{\theta}, \gamma)=\Psi\left(B(\bar{M}, \dot{\theta}, \gamma)+N, \tilde{d}_{r}, d_{\theta}+1, \tilde{n}\right)
$$

where $B(\bar{M}, \dot{\theta}, \gamma)$ is a multilinear function in $\tilde{M}$ and $\dot{\theta}$, i.e., it is linear in $\tilde{M}$ for fixed $\dot{\theta}$ and fixed $\gamma$, and is also linear in $\dot{\theta}$ for fixed $\tilde{M}$ and fixed $\gamma$, and $N$ is a symmetric matrix belonging to the set

$$
\mathscr{N}=\left\{N^{T}=N: \Psi\left(N, \tilde{d}_{r}, d_{\theta}+1, \tilde{n}\right)=0\right\} .
$$


Lemma 8: $\mathscr{N}$ is a linear space whose dimension is

$$
\begin{aligned}
\sigma\left(\tilde{n}, \tilde{d}_{r}, d_{\theta}+1\right) & =\frac{1}{2} \tilde{n}(l(\tilde{n} l+1) \\
& \left.-(\tilde{n}+1) l_{\mathrm{hom}}\left(a, 2 d_{\theta}+2\right) l_{\mathrm{pol}}\left(\tilde{n}, 2 \tilde{d}_{r}\right)\right)
\end{aligned}
$$

where $l=l_{\text {pol }}\left(\tilde{n}, \tilde{d}_{r}\right) l_{\text {hom }}\left(a, d_{\theta}+1\right)$.

Proof Similar to the proof of Lemma 7 and we omit it here.

For more details of SMR and complete parametrization of affine spaces, interested readers can refer [35], [36] and its developments in robust consensus and robust synchronization [37]-[39]. The following result gives a sufficient condition which is a convex problem of LMIs feasibility test.

Theorem 3: The robust exponential synchronization of (6) can be achieved under Assumption 1 if there exist matrices $\bar{M}(\alpha), N(\beta)$ and a positive scalar $\gamma$ such that,

$$
\left\{\begin{array}{l}
0<\bar{M}(\alpha) \\
0>B\left(\bar{M}(\alpha), d^{j}, \gamma\right)+N\left(\beta^{j}\right), \forall j=1, \ldots, v .
\end{array}\right.
$$

where $\bar{M}(\alpha)$ and $N(\beta)$ are linear parametrizations of affine spaces $\mathscr{M}$ and $\mathscr{N}$ respectively, and $\alpha, \beta^{j}$ are corresponding free parameters whose dimensions are given by Lemma 7 and Lemma 8 , for all $j=1, \ldots, v$.

Proof Let us consider the first inequality in (20), $\forall \theta \in \mathbb{R}_{0}^{a}$, by pre- and post-multiplying $\left(\phi_{\text {pol }}\left(y, d_{y}\right) \otimes \phi_{\text {hom }}\left(\theta, d_{\theta}\right) \otimes I_{\tilde{n}}\right)^{T}$ and $\left(\phi_{\text {pol }}\left(y, d_{y}\right) \otimes \phi_{\text {hom }}\left(\theta, d_{\theta}\right) \otimes I_{\tilde{n}}\right)$, one can obtain

$$
0<M(y, \operatorname{sq}(\theta)) .
$$

Similarly, from the second inequality in (20), $\forall y \in \mathbb{R}_{0}^{\tilde{n}}$ and $\forall(\theta, \dot{\theta}) \in \Omega$, by pre- and post-multiplying $\left(\phi_{\text {pol }}\left(y, \tilde{d}_{r}\right) \otimes\right.$ $\left.\phi_{\text {hom }}\left(\theta, d_{\theta}+1\right) \otimes I_{\tilde{n}}\right)^{T}$ and $\left(\phi_{\text {pol }}\left(y, \tilde{d}_{r}\right) \otimes \phi_{\text {hom }}\left(\theta, d_{\theta}+1\right) \otimes I_{\tilde{n}}\right)$, it follows that there exists a positive scalar $\gamma$ such that

$$
0>\Psi\left(B\left(\bar{M}, d^{j}, \gamma\right)+N\left(\beta^{j}\right), \tilde{d}_{r}, d_{\theta}+1, \tilde{n}\right), \forall j=1, \ldots, v .
$$

In addition, considering $N\left(\beta^{j}\right) \in \mathscr{N}$, one has

$$
\Psi\left(N\left(\beta^{j}\right), \tilde{d}_{r}, d_{\theta}+1, \tilde{n}\right)=0, \forall j=1, \ldots, v .
$$

Therefore, it follows that there exists a positive scalar $\gamma$ such that

$$
0>R(y, \operatorname{sq}(\theta), \dot{\theta}, \gamma),
$$

Since $\Xi$ is a convex hull of vectors $d^{j}$ for $j=1, \ldots, v$, the condition of Lemma 6 holds which completes the proof.

A paralleling result can be provided by the same approach for robust asymptotical contraction as follows:

Corollary 1: The robust asymptotical synchronization of (6) can be achieved under Assumption 1 if it satisfies following condition,

$$
\left\{\begin{array}{l}
0<\bar{M}(\alpha) \\
0>\widetilde{B}\left(\bar{M}(\alpha), d^{j}\right)+N\left(\beta^{j}\right), \forall j=1, \ldots, v,
\end{array}\right.
$$

where

$$
\widetilde{R}(y, \theta, \dot{\theta})=\Psi\left(\widetilde{B}(\bar{M}, \dot{\theta})+N, \tilde{d}_{r}, d_{\theta}+1, \tilde{n}\right),
$$

$$
\begin{aligned}
& \widetilde{R}(y, \theta, \dot{\theta}) \\
= & 2\left(\sum_{i=1}^{a} \theta_{i}\right)\left(\frac{\partial g^{T}}{\partial y} M\right)^{\mathrm{s}}-2 b\left((L(\theta) \otimes \Gamma)^{T} M\right)^{\mathrm{s}} \\
& +\left(\sum_{i=1}^{a} \theta_{i}\right)^{2}\left(\frac{\partial M^{T}}{\partial \theta} \dot{\theta}\right)^{\mathrm{s}}+\left(\sum_{i=1}^{a} \theta_{i}\right)\left(\frac{\partial M^{T}}{\partial y} g\right)^{\mathrm{s}} \\
& -b\left(\frac{\partial M}{\partial y}(L(\theta) \otimes \Gamma) y\right)^{\mathrm{s}} .
\end{aligned}
$$

Remark 5: It is useful to note that

- Considering the synchronization protocol

$$
\dot{x}_{i}=f\left(x_{i}, \theta\right)+\sum_{j=1}^{N} u_{i j}\left(x_{j}-x_{i}, \theta\right), \forall i=1, \ldots, N,
$$

where function $f$ is a polynomial in $x_{i}$ and $\theta$, and $u_{i j}\left(x_{j}-x_{i}, \theta\right)$ is also a polynomial in $\left(x_{j}-x_{i}\right)$ and $\theta$. For this general case, an approach of polynomial parameter-dependent polynomial contraction matrix can be provided, while (17) can be presented similarly as

$$
\widetilde{\Psi}\left(\bar{M}, d_{y}, d_{\theta}, \tilde{n}\right)=(*)^{T} \bar{M}\left(\phi_{\text {pol }}\left(y, d_{y}\right) \otimes \phi_{\text {pol }}\left(\theta, d_{\theta}\right) \otimes I_{\tilde{n}}\right) .
$$

- Theorem 3 and Corollary 1 provide tractable conditions for robust synchronization. However, it is admitted that the conservatism arises between Theorem 1 and Theorem 3 because of the gap between positive polynomials and Sum-of-Square polynomials which relates to the Hilbert's 17th problem [40].

\section{Robust Synchronization Performance}

Section III provides conditions on which the robust exponential or asymptotical synchronization with bounded-rate polytopic uncertainties can be achieved. Follow-up question arises naturally that what is the largest level of polytopic uncertainties on which the robustness of asymptotical synchronization maintains. This section gives the answer for this question.

Considering time-varying bounded-rate polytopic uncertainty given by (7), a variation rate margin of robust asymptotical synchronization can be defined for uncertain synchronization protocol (6). Let $\eta$ be variation rate margin for system (6) as follows:

$$
\begin{aligned}
\eta= & \sup \{\eta \in \mathbb{R}:(6) \text { achieves robust synchronization, } \\
& \left.\forall \dot{\theta} \in \operatorname{co}\left\{\eta d^{(1)}, \ldots, \eta d^{(v)}\right\}, \forall \theta \in \Lambda_{a}\right\} .
\end{aligned}
$$

It is of special usefulness that another definition comes from a typical instance of above denotation, which concerns on the cases that robust asymptotical synchronization is guaranteed by a HPD-PACM $M(y, \mathrm{sq}(\theta))$ given by (17) for system (6) as follows:

Definition 5: Define $\eta_{\left\{d_{y}, d_{\theta}\right\}}$ as $\left\{d_{y}, d_{\theta}\right\}$-HPD-PACM variation rate margin for system (6) if there exists a HPD-PACM $M(y, \theta)$ given by (17) for system (6) such that

$$
\begin{aligned}
\eta_{\left\{d_{y}, d_{\theta}\right\}}= & \sup \left\{\eta \in \mathbb{R}: \dot{\theta} \in \operatorname{co}\left\{\eta \mathrm{d}^{(1)}, \ldots, \eta \mathrm{d}^{(\mathrm{v})}\right\},\right. \\
& \left.\forall \theta \in \Lambda_{a}\right\} .
\end{aligned}
$$


Obviously, $\eta_{\left\{d_{y}, d_{\theta}\right\}}$ is a lower bound of the variation rate margin under the guarantee of robust asymptotical synchronization by the class of HPD-PACM. Specifically, one has

$$
\eta_{\left\{d_{y}, d_{\theta}\right\}} \leq \eta, \forall d_{y}, \forall d_{\theta} .
$$

The following results gives a strategy for obtaining a lower bound of $\eta_{\left\{d_{y}, d_{\theta}\right\}}$ by solving a GEVP problem.

Theorem 4: Let us define

$$
\hat{\eta}_{\left\{d_{y}, d_{\theta}\right\}}=\frac{1}{\varsigma^{*}}
$$

where $\varsigma^{*}$ is the solution of

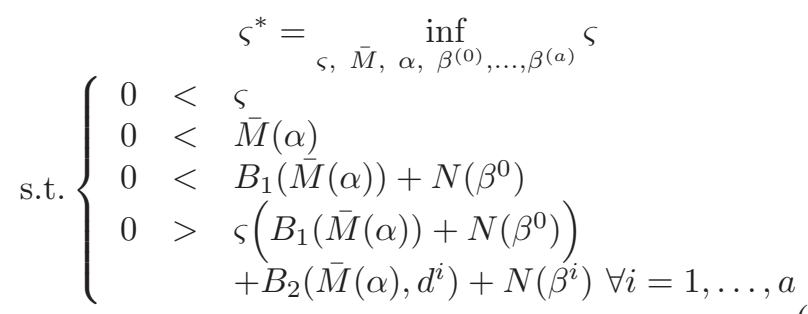

where $\bar{M}(\alpha)$ is a linear parametrization of space $\mathscr{M}, N(\beta)$ is a linear parametrization of space $\mathscr{N}, R(y, \theta, \dot{\theta})=R_{1}(y, \theta, \dot{\theta})+$ $R_{2}(y, \theta, \dot{\theta})$,

$$
\begin{aligned}
& R_{1}(y, \theta, \dot{\theta}) \\
= & 2\left(\sum_{i=1}^{a} \theta_{i}\right)\left(\frac{\partial g^{T}}{\partial y} M\right)^{\mathrm{s}}-2 b\left((L(\theta) \otimes \Gamma)^{T} M\right)^{\mathrm{s}} \\
& +\left(\sum_{i=1}^{a} \theta_{i}\right)\left(\frac{\partial M^{T}}{\partial y} g\right)^{\mathrm{s}}-b\left(\frac{\partial M}{\partial y}(L(\theta) \otimes \Gamma) y\right)^{\mathrm{s}}, \\
& R_{2}(y, \theta, \dot{\theta}) \\
= & \left(\sum_{i=1}^{a} \theta_{i}\right)^{2}\left(\frac{\partial M^{T}}{\partial \theta} \dot{\theta}\right)^{\mathrm{s}},
\end{aligned}
$$

and

$$
\begin{aligned}
& R_{1}(y, \theta, \dot{\theta})=\Psi\left(B_{1}(\bar{M})+N, \tilde{d}_{r}, d_{\theta}+1, \tilde{n}\right) \\
& R_{2}(y, \theta, \dot{\theta})=\Psi\left(B_{2}(\bar{M}, \dot{\theta})+N, \tilde{d}_{r}, d_{\theta}+1, \tilde{n}\right) .
\end{aligned}
$$

Then $\hat{\eta}_{\left\{d_{y}, d_{\theta}\right\}}$ is the lower bound of $\eta_{\left\{d_{y}, d_{\theta}\right\}}$, i.e. $\hat{\eta}_{\left\{d_{y}, d_{\theta}\right\}} \leq$ $\eta_{\left\{d_{y}, d_{\theta}\right\}} \cdot$

Proof Suppose that (25) holds. Pre- and post-multiplying the second inequality in (25) by $\left(\phi_{\text {pol }}\left(y, d_{y}\right) \otimes \phi_{\text {hom }}\left(\theta, d_{\theta}\right) \otimes I_{\tilde{n}}\right)^{T}$ and $\left(\phi_{\text {pol }}\left(y, d_{y}\right) \otimes \phi_{\text {hom }}\left(\theta, d_{\theta}\right) \otimes I_{\tilde{n}}\right)$, respectively, one has that

$$
0<\Psi\left(\bar{M}, d_{y}, d_{\theta}, \tilde{n}\right)
$$

hence implying $M(y, \theta)$ is positive definite since $\left(\phi_{\text {pol }}\left(y, d_{y}\right) \otimes \phi_{\text {hom }}\left(\theta, d_{\theta}\right) \otimes I_{\tilde{n}}\right)^{T}\left(\phi_{\text {pol }}\left(y, d_{y}\right) \otimes \phi_{\text {hom }}\left(\theta, d_{\theta}\right) \otimes\right.$ $\left.I_{\tilde{n}}\right)>0$ for all $y \neq 0$. Then, $R(y, \theta, \dot{\theta})$ for $\dot{\theta}=\varsigma^{-1} \nu^{(i)}$ is given by

$$
\begin{aligned}
& \left.R(y, \theta, \dot{\theta})\right|_{\dot{\theta}=\varsigma^{-1} \nu^{(i)}} \\
= & \Psi\left(B_{1}(\bar{M})+\varsigma^{-1} B_{2}\left(\bar{M}, d^{i}\right), \tilde{d}_{r}, d_{\theta}+1, \tilde{n}\right) \\
= & \varsigma^{-1} \Psi\left(\varsigma B_{1}(\bar{M})+B_{2}\left(\bar{M}, d^{i}\right), \tilde{d}_{r}, d_{\theta}+1, \tilde{n}\right) .
\end{aligned}
$$

since $N\left(\beta^{i}\right) \in \mathscr{N}, \forall i=0,1, \ldots, a$, it follows

$$
\begin{aligned}
& \left.R(y, \theta, \dot{\theta})\right|_{\dot{\theta}=\varsigma^{-1} \nu^{(i)}} \\
& =\varsigma^{-1} \Psi\left(\varsigma\left(B_{1}(\bar{M}(\alpha))+N\left(\beta^{0}\right)\right)\right. \\
& \left.+B_{2}\left(\bar{M}(\alpha), d^{i}\right)+N\left(\beta^{i}\right), \tilde{d}_{r}, d_{\theta}+1, \tilde{n}\right) .
\end{aligned}
$$

Thus, due to the last constraint in (25) one has

$$
\left.R(y, \theta, \dot{\theta})\right|_{\dot{\theta}=\varsigma^{-1} \nu^{(i)}}<0 \quad \forall i=1, \ldots, a .
$$

Based on this, one can also have that there exists a HPDPCM for all $\theta(t)$ in following set

$$
\begin{aligned}
\eta_{\left\{d_{y}, d_{\theta}\right\}=} & \sup \left\{\eta \in \mathbb{R}: \dot{\theta} \in \operatorname{co}\left\{\varsigma^{-1} d^{(1)}, \ldots, \varsigma^{-1} d^{(v)}\right\},\right. \\
& \left.\forall \theta \in \Lambda_{a}\right\} .
\end{aligned}
$$

Therefore, one has $\hat{\eta}_{\left\{d_{y}, d_{\theta}\right\}} \leq \eta$ which completes this proof.

\section{NUMERICAL EXAMPLES}

To illustrate our proposed approach, two deliberately simple examples are provided by using MATLAB and its toolboxes SMRSOFT and SeDuMi.

\section{A. Example 1}

In this example, a coupled model of Moore-Greitzer jet engines is considered in the no-stall mode [14], [41]. The intrinsic dynamics of each jet engine is described by $f(x)$ in (6) as

$$
f\left(x_{i}\right)=\left(\begin{array}{c}
-0.5 x_{i 1}^{3}-1.5 x_{i 1}^{2}-x_{i 2} \\
3 x_{i 1}-x_{i 2}
\end{array}\right)
$$

where $x_{i}=\left(x_{i 1}, x_{i 2}\right)^{\prime}, i=1,2, x_{i 1}$ relates to the mass flow and $x_{i 2}$ relates to the pressure rise. The communications between these two jet engines are disturbed by a time-varying uncertainty $\theta(t)$. Let the uncertain weighted adjacency matrix $G(\theta(t))$ be

$$
G(\theta(t))=\left(\begin{array}{cc}
1 & 0 \\
1-2 \theta(t) & 1
\end{array}\right) .
$$

For $\theta(t)>0.721$, the synchronization can not be achieved since a Hopf bifurcation takes place as shown in Fig. 1 where error states $z(t)=x_{1}(t)-x_{2}(t)$.In (a) of Fig. $1, \theta(t)=$ $0.6, \dot{\theta}=0$, synchronization can be achieved where trajectory of agent 1 is shown in (b). In (c) of Fig. $1, \theta(t)=0.75, \dot{\theta}=0$, synchronization can not be achieved where trajectory of agent 1 is shown in (d). Since $0_{a} \in \Xi=\operatorname{co}\left\{d^{(1)}, \ldots, d^{(v)}\right\}$, for any $\eta$ given by (23), the robust synchronization can not be achieved when $\theta(t)>0.721$. Hence in this example we consider the parameter bound $0 \leq \theta(t) \leq 0.6$.

Let $c=1, \Gamma=I_{2}$ and a maximum variation rate $\eta$ of $\theta(t)$ is considered such that the robust asymptotical synchronization can be achieved for any $|\dot{\theta}(t)| \leq \eta$. Hence, $\Xi$ can be expressed as

$$
\Xi=\operatorname{co}\left\{\left(\begin{array}{c}
\frac{\eta}{0.6} \\
-\frac{\eta}{0.6}
\end{array}\right),\left(\begin{array}{c}
-\frac{\eta}{0.6} \\
\frac{\eta}{0.6}
\end{array}\right)\right\} .
$$

TABLE I: Lower bound $\hat{\eta}$, for some values of $d_{y}$ and $d_{\theta}$.

\begin{tabular}{ccccc}
\hline \hline$d_{y} / d_{\theta}$ & 0 & 1 & 2 & 3 \\
\hline 1 & N/A & N/A & N/A & N/A \\
2 & 178.3 & 197.1 & 207.7 & 214.1 \\
3 & 185.8 & 202.3 & 211.2 & 216.4 \\
\hline
\end{tabular}


Similar to former example, we compute the lower bound $\hat{\eta}$ by employing HPD-PCM method with $d_{y}=1$ and $d_{\theta}=$ $0,1,2,3$ as shown in Table II. Comparing with sufficient conditions provided by [29] and by [14], again, the proposed method is testified to be less conservative and obtains a larger robust asymptotical synchronization margin with $d_{\theta}>1$. Furthermore, it also shows that by increasing the degree of uncertain parameter $d_{\theta}$, the conservatism level decreases progressively and apparently.

It is worthy to note that, comparing with approach of [29], even though same $d_{\theta}$ is considered, proposed method still obtains a bigger margin $\hat{\eta}$ (also shown in Table II) in that this paper completely parametrized corresponding affine spaces while [29] does not.

\section{CONCLUSIONS}

Robust synchronization of multi-agent system with polynomial nonlinear dynamics is considered affected by timevarying polytopic uncertainty with bounded variation rate. Based on partial contraction, a novel approach is proposed by using a new class of contraction matrix, i.e., homogeneous parameter-dependent polynomial contraction matrix (HPDPCM), and conditions for robust exponential synchronization and robust asymptotical synchronization are both provided. Corresponding sufficient conditions have also been proposed in terms of LMIs via exploring the parametrizations of related affine sets. Moreover, we investigate the variation rate for robust asymptotical synchronization margin whose lower bound can be estimated via solving GEVPs.

Comparing with Parameter Linear-dependent Quadratic Lyapunov Function (PLD-QLF) and parameter-independent polynomial contraction matrix, numerical examples have shown that the proposed method generalize above methods and can successfully decrease the conservatism level by using a higher-order HPD-PCM, in other words, an expanded lower bound of variation rate margin can be obtained via increasing the value of $d_{y}$ and $d_{\theta}$ respectively.

Future efforts will be devoted to investigate the upper bound of degree $d_{y}$ and $d_{\theta}$ to ensure the robust synchronization. In addition, particular interests have casted on stochastic synchronization with randomly switching topology, like representative stochastic models used in [15], [16], [18].

\section{ACKNOWLEDGMENT}

The authors would like to thank the Editors and the Reviewers for their valuable suggestions and comments. This work is supported in part by the Research Grants Council of Hong Kong under Grant HKU711213E.

\section{APPENDIX}

\section{A. Proof of Proposition 1}

From (3), one obtains

$$
|\delta x|_{q} \leq\left|\delta x_{0}\right|_{q} e^{\int_{0}^{t} \mu_{q}(J(\tau)) d \tau} .
$$

From Definition 1, the contraction of System 1 for $|\cdot|_{q}$ yields to

$$
|\delta x|_{q} \leq\left|\delta x_{0}\right|_{q} e^{-c_{q}}
$$

Clearly, one can obtain the contraction rate $c_{q}$ from the upper bound of matrix measure of system Jacobian as

$$
c_{q}=-\max \left\{\mu_{q}(J)\right\} .
$$

According to the equivalence between $|\cdot|_{q}$ and $|\cdot|_{p}$ from Lemma 2, one has

$$
|\delta x|_{p} \leq n^{(1 / q-1 / p)}\left|\delta x_{0}\right|_{p} e^{-c_{q} t}
$$

which can be alternatively expressed as

$$
|\delta x|_{p} \leq\left|\delta x_{0}\right|_{p} e^{-c_{q}(t-\psi)}
$$

where $\psi=\frac{(p-q) \log n}{p q c_{q}}$ denotes a time-shift.

\section{B. Proof of Lemma 7}

Let $\bar{M}_{1}$ and $\bar{M}_{2}$ be any matrices in $\mathscr{M}$. It directly follows that for any linear combination of $\bar{M}_{1}$ and $\bar{M}_{2}$, one has $c_{1} \bar{M}+$ $c_{2} \bar{M}_{2} \in \mathscr{M}$, for all $c_{1}, c_{2} \in \mathbb{R}$ such that $c_{1}+c_{2}=1$. Thus, one can obtain that $\mathscr{M}$ is an affine space.

Define

$$
a=\tilde{n} l_{\mathrm{pol}}\left(\tilde{n}, d_{y}\right) l_{\mathrm{hom}}\left(a, d_{\theta}\right),
$$

the total number of free entries of $\bar{M} \in \mathbb{R}^{a \times a}$ can be calculated as $\frac{1}{2} a(a+1)$. Let $b \in \mathbb{R}^{\frac{1}{2} a(a+1)}$ be a vector containing the free entries of matrix $\bar{M}$, and define a linear mapping $E$ : $\mathbb{R}^{\frac{1}{2} a(a+1)} \rightarrow \mathbb{R}^{a \times a}$ satisfying $E(b)=\bar{M}$. Thus, one has

$$
\begin{aligned}
& \Psi\left(\bar{M}, d_{y}, d_{\theta}, \tilde{n}\right) \\
& =(*)^{T} E(b)\left(\phi_{\mathrm{pol}}\left(y, d_{y}\right) \otimes \phi_{\mathrm{hom}}\left(\theta, d_{\theta}\right) \otimes I_{\tilde{n}}\right) \\
& =(F b)^{T}\left(\phi_{\mathrm{pol}}\left(y, 2 d_{y}\right) \otimes \phi_{\mathrm{hom}}\left(\theta, 2 d_{\theta}\right) \otimes I_{\tilde{n}}\right)
\end{aligned}
$$

where $F$ is a suitable transformation matrix. Observe that

$$
\mathscr{M}=\{E(b): b \in \operatorname{ker}(E)\} .
$$

It directly follows that

$$
\begin{aligned}
\operatorname{dim}(\mathscr{M}) & =\operatorname{dim}(\{E(b): b \in \operatorname{ker}(E)\}) \\
& =\operatorname{dim}(\operatorname{ker}(E)) \\
& =\frac{1}{2} a(a+1)-\operatorname{rank}(E) .
\end{aligned}
$$

Let us observe that dimension of $\mathscr{M}$ stems from the entries of same monomials with even power in $\theta$, implying that

$$
\begin{aligned}
\operatorname{rank}(E)= & \{\text { number of distinct monomials } \\
& \left.c_{i, j} \theta^{i} y^{j} \text { with odd power } i^{k}\right\} .
\end{aligned}
$$

For scalar homogeneous polynomial in $\theta$, the number of distinct monomials with at least one odd power of $\theta$ is $l_{\text {hom }}\left(a, 2 d_{\theta}\right)-l_{\text {hom }}\left(a, d_{\theta}\right)$. Considering all the distinct monomials in $y$ of degree less or equal to $d_{y}$ and in the matrix form with size $\tilde{n} \times \tilde{n}$, the total number of distinct monomials with at least one odd power of $\theta$ can be expressed as $\frac{1}{2} \tilde{n}(\tilde{n}+$ $1)\left(l_{\text {hom }}\left(a, 2 d_{\theta}\right)-l_{\text {hom }}\left(a, d_{\theta}\right)\right) l_{\mathrm{pol}}\left(\tilde{n}, 2 d_{y}\right)$, which completes the proof. 
[1] L. M. Pecora and T. L. Carroll. Synchronization in chaotic system. Physical Review Letters, 64(8):1146-1152, 1990.

[2] C. W. Wu. Synchronization in Coupled Chaotic Circuits and Systems. World Scientific, 2002.

[3] Z. Li, Z. Duan, G. Chen, and L. Huang. Consensus of multi-agent systems and synchronization of complex networks: A unified viewpoint. IEEE Transactions on Circuits and Systems-I, 57(1):213-224, 2010.

[4] J. Lü, G. Chen, X. Yu, and H. Leung. Design and analysis of multiscroll chaotic attractors from saturated function series. IEEE Transactions on Circuits and Systems-I, 51(12):2476-2490, 2004.

[5] J. Lü, X. Yu, G. Chen, and D. Cheng. Characterizing the synchronizability of small-world dynamical networks. IEEE Transactions on Circuits and Systems-I, 51(4):787-796, 2004.

[6] L. M. Pecora and T. L. Carroll. Master stability functions for synchronized coupled systems. Physical Review Letters, 80(10):2109-2112, 1998.

[7] L. M. Pecora. Synchronization in small-world systems. Physical Review E, 58(1):347-360, 1998.

[8] W. Lohmiller and J. J. E. Slotine. On contraction analysis for non-linear systems. Automatica, 34(6):683-696, 1998.

[9] W. Wang and J. J. E. Slotine. On partial contraction analysis for coupled nonlinear oscillators. Biological Cybernetics, 92(1):38-53, 2005.

[10] B. S. Chen, C. H. Chiang, and S. K. Nguang. Robust $\mathrm{H}_{\infty}$ synchronization design of nonlinear coupled network via fuzzy interpolation method. IEEE Transactions on Circuits and Systems-I, 58(2):349-362, 2011.

[11] B. Liu, X. Liu, G. Chen, and H. Wang. Robust impulsive synchronization of uncertain dynamical networks. IEEE Transactions on Circuits and Systems I: Regular Papers, 52(7):1431-1441, July 2005.

[12] D. J. Stilwell, E. M. Bollt, and D. G. Roberson. Sufficient Conditions for Fast Switching Synchronization in Time-Varying. SIAM Journal of Applied Dynamical Systems, 5(1):140-156, 2006.

[13] R. E. Amritkar and C.-K. Hu. Synchronized state of coupled dynamics on time-varying networks. Chaos, 16(1):015117, March 2006.

[14] E. M. Aylward, P. A. Parrilo, and J. J. E. Slotine. Stability and robustness analysis of nonlinear systems via contraction metrics and SOS programming. Automatica, 44(8):2163-2170, 2008.

[15] I. Belykh, V. Belykh, and M. Hasler. Blinking model and synchronization in small-world networks with a time-varying coupling. Physica $D$ : Nonlinear Phenomena, 195(1-2):188-206, August 2004.

[16] M. Porfiri, D. J. Stilwell, E. M. Bollt, and J. D. Skufca. Random talk: Random walk and synchronizability in a moving neighborhood network. Physica D: Nonlinear Phenomena, 224(1-2):102-113, 2006.

[17] M. Porfiri and R. Pigliacampo. Master-Slave Global Stochastic Synchronization of Chaotic Oscillators. SIAM Journal on Applied Dynamical Systems, 7(3):825-842, January 2008.

[18] M. Hasler, V. Belykh, and I. Belykh. Dynamics of Stochastically Blinking Systems. Part I: Finite Time Properties. SIAM Journal on Applied Dynamical Systems, 12(2):1007-1030, January 2013.

[19] M. Hasler, V. Belykh, and I. Belykh. Dynamics of Stochastically Blinking Systems. Part II: Asymptotic Properties. SIAM Journal on Applied Dynamical Systems, 12(2):1031-1084, January 2013.

[20] V. Belykh, I. Belykh, and M. Hasler. Connection graph stability method for synchronized coupled chaotic systems. Physica D: Nonlinear Phenomena, 195(1-2):159-187, August 2004.

[21] R. Carli and A. Chiuso. Optimal synchronization for networks of noisy double integrators. IEEE Transactions on Automatic Control, 56(5):1146-1152, 2011.

[22] V. I. Arnold. Mathematical Methods of Classical Mechanics. SpringerVerlag, 1978.

[23] W. A. Coppel. Stability and Asymptotic Behavior of Differential Equations. Heath, 1965.

[24] J. Jouffroy and J. J. E. Slotine. Methodological remarks on contraction theory. In Proceedings of the 43th IEEE Conference on Decision and Control, volume 3, pages 2537-2543, 2004.

[25] M. Vidyasagar. Nonlinear Systems Analysis, 2nd ed. Prentice-Hall, 1993.

[26] C. A. Desoer and M. Vidyasagar. Feedback Systems: Input-output Properties. Academic Press, 1975.

[27] L. P. Kuptsov. Hölder Inequality. Springer, 2001

[28] J. C. Geromel and P. Colaneri. Robust stability of time varying polytopic systems. Systems and Control Letters, 55(1):81-85, 2005. stability of linear time-varying systems. In Proceedings of the 42th IEEE Conference on Decision and Control, pages 6133-6138, Maui, Hawaii, U.S.A., 2003. Ieee.

[30] P. Gahinet, P. Apkarian, and M. Chilali. Affine parameter-dependent Lyapunov functions and real parametric uncertainty. IEEE Transactions on Automatic Control, 41(3):436-442, 1996.

[31] R. A. Horn and C. R. Johnson. Matrix Analysis. Cambridge University Press, 1985

[32] N. Aghannan and P. Rouchon. An intrinsic observer for a class of lagrangian systems. IEEE Transactions on Automatic Control, 48(6):936-945, 2003

[33] H. K. Khalil. Nonlinear Systems, 3rd ed. Prentice Hall, 2002.

[34] W. Yu, P. DeLellis, G. Chen, M. di Bernardo, and J. Kurths. Distributed adaptive control of synchronization in complex networks. IEEE Transactions on Automatic Control, 57(8):2153-2158, 2012.

[35] G. Chesi, A. Garulli, A. Tesi, and A. Vicino. Homogeneous Polynomial Forms for Robustness Analysis of Uncertain Systems. Springer-Verlag, 2009.

[36] G. Chesi, A. Garulli, A. Tesi, and A. Vicino. Polynomially parameterdependent Lyapunov functions for robust stability of polytopic systems: an LMI approach. IEEE Transactions on Automatic Control, 50(3):365$370,2005$.

[37] D. Han, G. Chesi, and Y. S. Hung. Robust consensus for a class of uncertain multi-agent dynamical systems. IEEE Transactions on Industrial Informatics, 9(1):306-312, 2013.

[38] D. Han and G. Chesi. Robust consensus for uncertain multi-agent systems with discrete-time dynamics. International Journal of Robust and Nonlinear Control, Jan. 2013. DIO:10.1002/rnc.2968.

[39] D. Han and G. Chesi. Synchronization conditions for multi-agent systems with intrinsic nonlinear dynamics. IEEE Transactions on Circuits and Systems-II, 60(4):227-231, 2013.

[40] G. Chesi. LMI techniques for optimization over polynomials in control: a survey. IEEE Transactions on Automatic Control, 55(11):2500-2510, 2010.

[41] M. Krstic, P. V. Kokotovic, and I. Kanellakopoulos. Nonlinear and Adaptive Control Design. John Wiley \& Sons, Inc., New York, NY, USA, 1st edition, 1995.

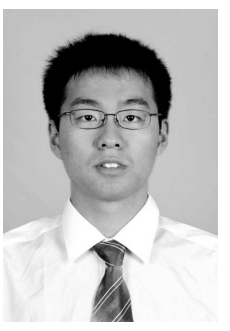

Dongkun Han is currently working toward the Ph.D. degree in control systems, Department of Electrical and Electronic Engineering, University of Hong Kong, Hong Kong. He was a visiting scholar of Peking University from Dec. 2009 to Feb. 2010 and a visiting research student of Stanford University from Jul. 2013 to Jan. 2014. His research interests include synchronization problem, robust control, convex optimization, complex network and cooperative control of multi-agent systems.

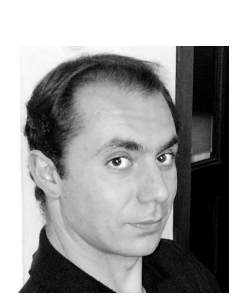

Graziano Chesi received the Laurea in Information Engineering from the University of Florence in 1997 and the Ph.D. in Systems Engineering from the University of Bologna in 2001. He was with the Department of Information Engineering of the University of Siena during 2000-2006 and then he joined the Department of Electrical and Electronic Engineering of the University of Hong Kong. Dr. Chesi has served as Associate Editor for Automatica, BMC Research Notes, the European Journal of Control, the IEEE Transactions on Automatic Control, and Systems \& Control Letters. Also, he has served as Guest Editor of the Special Issues on Positive Polynomials in Control, Systems Biology, and Visual Servoing for various journals. He is the Founder and Chair of the Technical Committee on Systems with Uncertainty of the IEEE Control Systems Society. Dr. Chesi is author of 3 books and first author in more than 100 publications. He is in the Top $1 \%$ Scholars of the University of Hong Kong. 